\title{
PERFORMANCE EVALUATION OF A LIGHT-WEIGHT MULTI-ECHO LIDAR FOR UNMANNED ROTORCRAFT APPLICATIONS
}

\author{
G. Conte, A. Kleiner, P. Rudol, K. Korwel, M. Wzorek, P. Doherty \\ Artificial Intelligence and Integrated Computer System Division \\ Linköping University \\ SE-581 83 Linköping, SWEDEN \\ gianpaolo.conte@liu.se \\ http://www.ida.liu.se/divisions/aiics/aiicssite/uastech/index.en.shtml
}

\begin{abstract}
KEY WORDS: UAS, LiDAR, Sensor Fusion, Inertial Sensors, Photogrammetry
\end{abstract}
\begin{abstract}
:
The paper presents a light-weight and low-cost airborne terrain mapping system. The developed Airborne LiDAR Scanner (ALS) system consists of a high-precision GNSS receiver, an inertial measurement unit and a magnetic compass which are used to complement a LiDAR sensor in order to compute a digital surface model. Evaluation of the accuracy of the generated surface model is presented. Additionally, a comparison is provided between the surface model generated from the developed ALS system and a model generated using a commercial photogrammetric software. Finally, the multi-echo capability of the used LiDAR sensor is evaluated in areas covered with dense vegetation.

The ALS system and camera systems were mounted on-board an industrial unmanned helicopter of around 100 kilograms maximum take-off weight. Presented results are based on real flight-test data.

\section{INTRODUCTION}

The development of Unmanned Aircraft Systems (UAS) has gained tremendous momentum over the last two decades. These platforms offer many advantages over both manned aircraft and other types of autonomous robots. They provide the ability to gather rich bird's eye view information in areas that may not be easily accessible to ground vehicles. Because of their mobility they also provide fast response times which is crucial in many applications such as search and rescue. A UAS can operate in hazardous environments, for example military conflicts or natural catastrophes,

The ALS has been integrated with the UAS Tech Lab RMAX helicopter (Section 3) and tested in real flights. The obtained results are evaluated with focus on accuracy in modeling of building structures and effectiveness of multi-echo technology in vegetation penetration. In addition, the paper presents a comparison between digital elevation models of a small airfield with a number of building structures. The models were generated using the presented ALS system and from a photogrammetric commercial software named Pix4UAV Desktop 3D 2.1 (Pix4d, 2013). The input to the software is a set of geotagged images generated by a consumer grade camera mounted on the UAV platform.
\end{abstract} without endangering the lives of human pilots or operators.

The work presented in this paper focuses on the terrain map building functionalities required in scenarios such the one defined by a new EU IP SHERPA Project (Smart collaboration between Humans and ground-aErial Robot for imProving rescuing activities in Alpine environments, (Marconi et al., 2012)). The goal of the SHERPA project is to develop a team of heterogeneous robotic platforms (i.e. ground and aerial) for supporting search and rescue activities in the real-world hostile environments such as in alpine rescue missions. The main task for the robotic system in this context is to enhance the limited perceptive capability of a team of rescuers. The ability to generate 3D models of a terrain covered with snow is therefore instrumental to achieving the mission goals.

Airborne LiDAR Scanner (ALS) systems have been an active research area during the past two decades. Since their introduction in the mid-90s the technology has developed substantially in terms of increased accuracy, increased pulse repetition rate, introduction of multiple returns (including full waveform digitizing) and integration with a higher accuracy GNSS and inertial navigation systems.

This paper presents a low-cost and light-weight ALS system and its evaluation for close range airborne applications. The presented ALS is suited for small and mid-size UAVs for applications where a terrain map building functionalities are required such as the search and rescue applications mentioned previously.

\section{RELATED WORK}

Mapping using helicopters is inherently difficult due to heavy vibrations that might occur during the flight. Consequently, range measurements related to pose-estimation are typically erroneous and conventional methods for point cloud registration might fail in certain cases. Kaestner et al. proposed a probabilistic approach for alignment and change detection using range sensor data (Kaestner et al., 2006), which they evaluated on a helicopter platform. They introduced non-rigid point cloud registration that implicitly deals with high estimation errors.

Recently, several small scale systems for mapping have been proposed (Glennie et al., 2013, Lin et al., 2011). A mobile laser scanning system for post-mission map generation from raw data that has been recorded by various sensors such as GNSS, INS, and laser scanner, was presented by (Glennie et al., 2013). The system is comparable to our approach, however, it is mainly tailored to be operated from a backpack or a balloon where it achieves a vertical accuracy of up to $3 \mathrm{~cm}$ and an horizontal accuracy of $17 \mathrm{~cm}$. A laser mapping system for small helicopters has been developed by Lin et al. (Lin et al., 2011). The authors demonstrated the feasibility of their approach by processing data from manual navigation.

Laser-based mapping has also already been evaluated in the context of avalanche prediction. Vallet and colleagues introduced the 


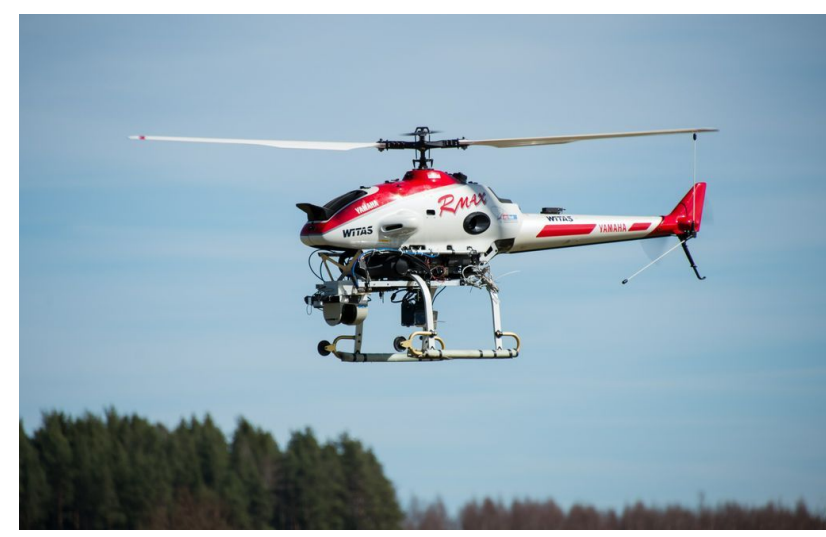

Figure 1: The UAS Tech Lab RMAX helicopter.

Helimap system developed by the photogrammetric and geodetic laboratories of the Swiss Federal Institute of Technology in Lausanne (EPFL) that was built for measuring snow volumes of large avalanche experimental fields (Vallet, 2002, Vallet, 2008). While the system achieved an accuracy of about $10 \mathrm{~cm}$, it has been designed mainly for manual operation on manned helicopters. Williams et al. proposed a vision-based SLAM algorithm for monitoring elevation changes in glacial regions (Williams et al., 2012). Their system was tailored to meet the challenges of low contrast glacial environments. Both the robots pose estimate and the positions of a large number of terrain landmark points are computed by the system. The presented solution makes use of Gaussian process (GP) regression to combine sparse visual landmarks extracted from the glacial scenery into a dense topographic map.

LiDAR systems have been extensively used also for forestry applications (Pirotti, 2011) in order to estimate key forest structure characteristics such as canopy height, topography, average stem diameter, canopy volume etc.

\section{HELICOPTER PLATFORM}

The platform used for experimentation presented in this paper is the UAS Tech Lab (UASTL) RMAX. It is a modified Yamaha RMAX helicopter (Figure 1). It uses a $21 \mathrm{hp} \mathrm{two-stroke} \mathrm{engine,}$ it has an empty weight of $61 \mathrm{~kg}$ and a maximum takeoff weight of $95 \mathrm{~kg}$. The main rotor diameter is 3.1 meters.

The UASTL RMAX is capable of autonomous flight from takeoff to landing. The hardware and software enabling the autonomous flight capabilities was developed at the Division of Artificial Intelligence and Integrated Computer System (AIICS) at Linköping University (Doherty et al., 2004, Conte, 2009).

\section{AIRBORNE LIDAR SYSTEM DESCRIPTION}

The following subsections describe the hardware components and algorithms used in the proposed Airborne LiDAR System.

\subsection{Hardware}

The ALS described in this paper is depicted in Figure 2. The system consists of a SICK LMS511 PRO $^{1}$ LiDAR with a range of 65 meters and capability of recording 5 echoes per one laser pulse. The TRE-G3T Javad RTK GNSS positioning system is used. The ground reference correction signal is transmitted to the

\footnotetext{
${ }^{1} \mathrm{http}: / /$ www.sick.com
}

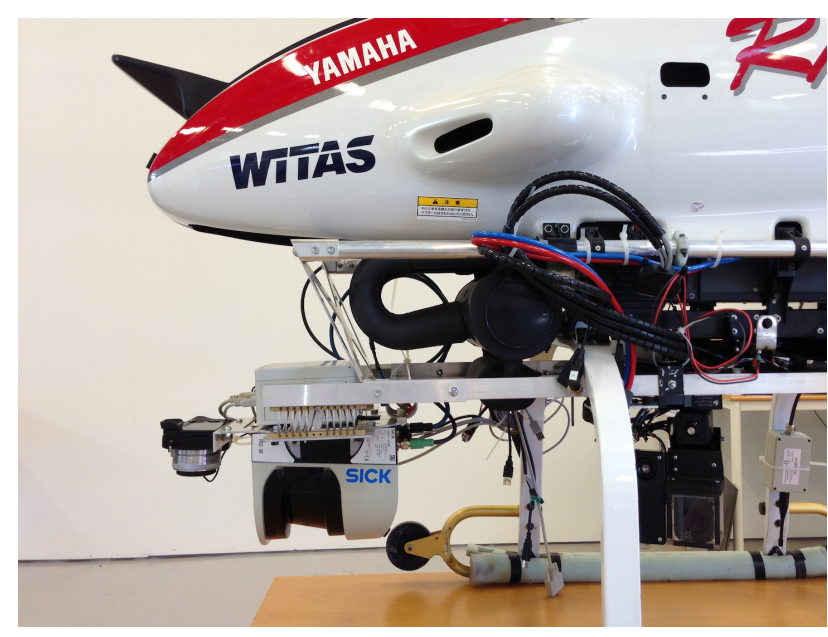

Figure 2: The Airborne LiDAR and camera systems mounted on the UASTL RMAX system.

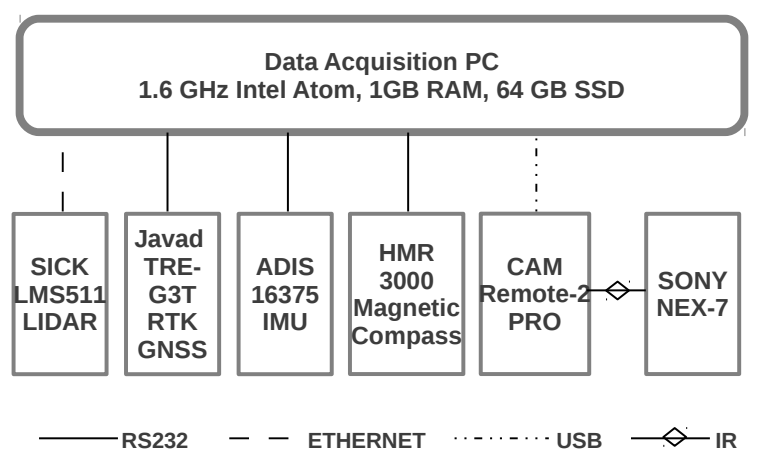

Figure 3: Schematics of the ALS and photogrammetric hardware components.

on-board receiver using the Telit TinyOne Plus ${ }^{2} 868 \mathrm{MHz}$ wireless datalink. An Analog Devices ADIS16375 MEMS Inertial Measurement Unit (IMU) and a Honeywell HMR3000 magnetic compass are additional parts of the system. An embedded PC104 1.6 Ghz Intel Atom computer is used for on-board data acquisition. The schematic of the system is presented in Figure 3 (the picture includes also the camera system for photogrammetric reconstruction described in section 5). The main characteristics of the sensors used in the proposed ALS are presented in Table 4.1.

The LiDAR and IMU are mounted on a vibration isolated rig in front of the UASTL RMAX platform (see Figure 2). The data acquisition PC and the GNSS receiver are mounted on a vibration isolated plate on a side of the UAV. The magnetic compass is placed on the helicopter tail boom to minimize magnetic interferences influencing the measurements.

Compared to high-end ALS traditionally used in the remote sensing field, the system presented here has a much lower cost (approximately $€ 15 \mathrm{~K}$ versus $€ 200 \mathrm{~K}$ for high-end systems). Additionally, it is lighter but has lower accuracy. The accuracy problem must be placed in the context of the intended usage of the system. The target use for the proposed ALS is for small and medium sized Unmanned Aerial Vehicles flying at low altitudes of 50-100 meters Above Ground Level (AGL). Typically, highend ALSs installed on manned aircrafts operate at altitudes of 500 meters AGL or more. Considering that direct georeferencing errors (direct georeferencing is a method used to produce point clouds from ALS measurements (Skaloud, 1999)) increase when

\footnotetext{
${ }^{2}$ http://www.telit.com
} 


\begin{tabular}{|c|c|c|c|c|}
\hline Sensor & \multicolumn{4}{|c|}{ Tech. Spec. } \\
\hline SICK Laser & Weight & Data Rate & Range & Echoes \\
\hline LMS511 PRO & $3.7 \mathrm{~kg}$ & $28.6 \mathrm{kHz}$ & $65 \mathrm{~m}$ & 5 \\
\hline Javad GNSS & & & Pos. Err. & RTK \\
\hline TRE-G3T & $77 \mathrm{~g}$ & $20 \mathrm{~Hz}$ & $5 \mathrm{~cm}$ & yes \\
\hline AirAnt antenna & $320 \mathrm{~g}$ & & & \\
\hline IMU & & & Gyro bias & Acc. bias \\
\hline ADIS16375 & $25 \mathrm{~g}$ & $100 \mathrm{~Hz}$ & $12 \mathrm{deg} / \mathrm{hr}$ & $0.13 \mathrm{mg}$ \\
\hline Compass & & & Accuracy & \\
\hline HMR3000 & $92 \mathrm{~g}$ & $15 \mathrm{~Hz}$ & $<1.5^{\circ}$ & \\
\hline $\begin{array}{l}\text { Sensor fusion } \\
\text { accuracy }\end{array}$ & $\begin{array}{r}\text { Position } \\
5 \mathrm{~cm}\end{array}$ & $\begin{array}{r}\text { Pitch/Roll } \\
<0.3^{\circ}\end{array}$ & $\begin{array}{r}\text { Heading } \\
<1.5^{\circ}\end{array}$ & \\
\hline
\end{tabular}

Table 1: Airborne LiDAR system sensor specification.

increasing the AGL altitude it can be reasonable to assume that, from an accuracy point of view, low cost ALS flying at low altitudes can be compared to high-end ALS flying at higher altitudes.

\subsection{Data Acquisition}

The raw data produced by the sensors described in the previous section is saved by data acquisition PC on a Solid State Drive (SSD). The software developed for the purpose of this work collects timestamped data using the appropriate interfaces (see Figure 3) and saves it in text files. Even though a non-realtime Linux OS is used, no negative consequences of it have been observed during the system operation.

\subsection{Processing}

The data processing pipeline consists of the following steps. Synchronized sensor data collected during a flight, post-processing of the IMU, RTK GNSS and compass data using an efficient twopass Kalman filter based smoothing algorithm (RauchTungStriebel smoother (Rauch et al., 1965)) and finally direct georeferencing of LiDAR measurements and point cloud generation.

\section{PHOTOGRAMMETRY SYSTEM DESCRIPTION}

In order to validate the ALS described in the previous section, a photogrammetry system has been built. The following subsections describe the components and algorithms used for the evaluation.

\subsection{Hardware}

The camera used in the system is the Sony Nex-7 and it is mounted on a vibration isolated rig in front the of the UASTL RMAX platform (see Figure 2) pointing downward. It features a 24 megapixel APS-C CMOS imaging sensor, a mirrorless construction and allows for changing lenses. The weight of the camera including the lens is approximately $0.5 \mathrm{~kg}$. The camera interfaces with the data acquisition $\mathrm{PC}$ using the CAMremote- $2 \mathrm{PRO}^{3}$ infrared (IR) interface. It allows for setting many of the parameters as well as triggering image acquisition from software. The physical connection schematics is shown in Figure 3.

\subsection{Data Acquisition}

Proper flight planning is needed for the phtogrammetric software in order to work properly. Particular care must be taken to ensure that the pictures collected have a sufficiently large overlapping region. The flight pattern which is typically used to scan a region is a regular rectangular pattern. Flight speed, lateral distance between consecutive scanning lines, flight altitude, camera focal

\footnotetext{
${ }^{3}$ http://vp-systems.eu/camremote.html
}

length, image acquisition rate and image resolution are sensitive parameters which must be accurately chosen. The time of triggering of the image capture is logged and used for synchronization with the GNSS data. Images are saved on the camera's memory card and downloaded afterwards for processing.

\subsection{Processing}

As already mentioned, the processing is done with Pix4UAV Desktop 3D 2.1 (Pix4d, 2013) software. The software allows to use either ground control points or geotagged images. The second option was used in this paper as the GNSS position is anyway available and used for the LiDAR system. Image geotagging information is added in the Exchangeable Image File (EXIF) metadata. It must be pointed out that the images were geotagged using only GNSS position information. No attitude information is used in the photogrammetric processing therefore the solution is not based on the direct georeferencing method but purely on computer vision techniques. When the processing is finished, the software offers the option to manually refine the results by adjusting or removing artifacts using a graphical user interface.

\section{EXPERIMENTAL RESULTS}

This section presents terrain reconstruction results using the ALS described in section 4 for two different landscapes: a small airfield with a few building structures and a tree covered field. In addition, the reconstruction of the airfield is performed using the photogrammetric system described in section 5 and the results are compared to the model generated with the ALS. Photogrammetric results for the tree-covered field are not presented here as this technique presents some complications in this kind of environment.

\subsection{Airfield Mapping}

The airfield object of this study is located near Motala (Sweden). Only the area with the building structures has been reconstructed. An overview of the area is depicted in Figure 4.

The size of the mapped area is about 1 ha. The flight was performed in a rectangular scanning pattern consisting of 3 flight lines with a separation of roughly 40 meters between them. The flight altitude was approximately 45 meters and the flight speed 3 $\mathrm{m} / \mathrm{s}$. LiDAR and camera data were acquired simultaneously. The LiDAR scanning angle was set at $90 \mathrm{deg}$ with a resolution of 0.66 deg giving an overlap between consecutive LiDAR strips of $57 \%$. The LiDAR scanning rate was set at $100 \mathrm{~Hz}$ per scanning line. Given the flight parameters and the LiDAR configuration parameters, the ground point density of a single strip can be calculated and it is about 50 points per square meter.

For what concerns the photogrammetric system the camera image size was set at $3008 \times 2000$ pixels and a $16 \mathrm{~mm}$ fixed focal lens was used. With such settings we get a ground resolution of $20 \mathrm{~cm} / \mathrm{pixel}$, a picture frontal overlap of $86 \%$ (along the flight direction) and a side overlap of $40 \%$.

Figure 5 (a) shows a snapshot of the airfield surface model generated with the ALS. The surface model is composed of three consecutive overlapping strips. A slight offset between the overlapping parts of the strips has been observed. This is due to systematic errors of the LiDAR system (Huising and Pereira, 1998, Skaloud and Lichti, 2006, Habib et al., 2008). Such errors are mainly caused by biases in the boresight parameters (mounting angle between the IMU sensor and the LiDAR sensor), errors in the lever arm correction between the different sensors, biases in 


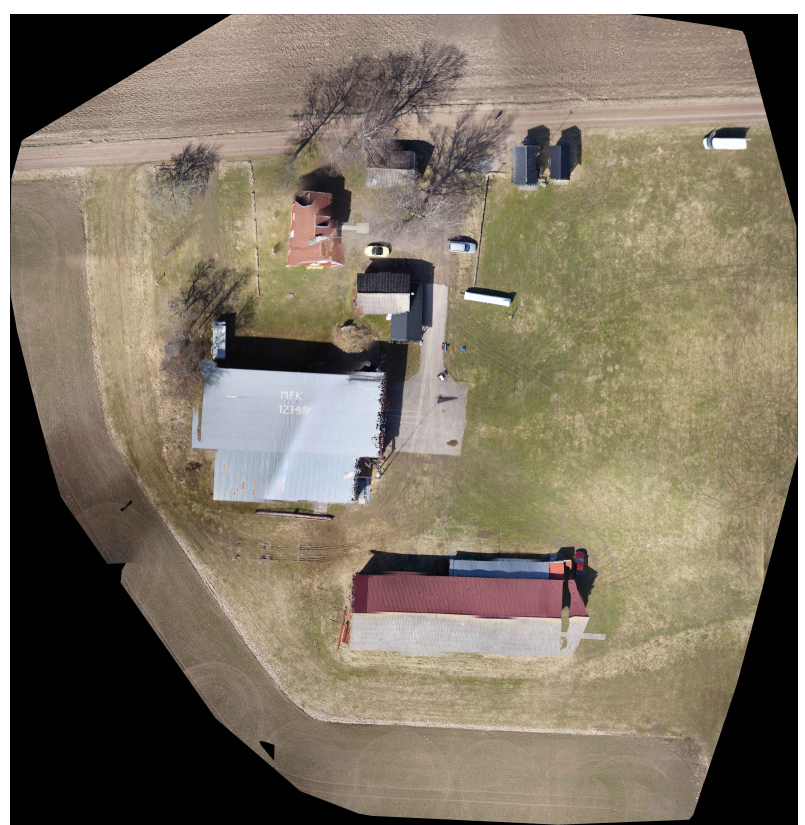

(a) Reconstructed orthomosaic.

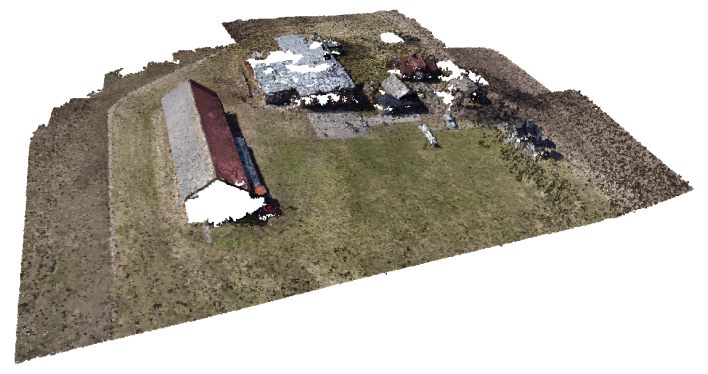

(b) Textured surface model.

Figure 4: Orthomosaic and textured surface model of the airfield in Motala (Sweden) object of the reconstruction analysis. The orthomosaic and the surface model are generated with the photogrammetric software Pix4UAV.

the LiDAR scanner device (ranges and mirror angle) and biases in the estimated attitude angle from IMU/GNSS/COMPASS integration.

The photogrammetric model was generated from 76 images. No ground control points were used during the processing with Pix 4 D software. Instead, the images were geotagged. At the first attempt only 6 images have been geotagged with poor results. The number of geotagged images has been increased until all images were geotagged for the final result shown in Figure 5 (b). The images were geotagged with high accuracy RTK GNSS position.

From a visual inspection of Figure 5 (a) and (b) it can be observed that neither the ALS nor the photogrammetric models are complete. Some parts of the buildings are missing in both models. It can be observed that the photogrammetric model is slightly more noisy than the ALS one. In addition, on the right side of the reconstructed model there is photogrammetric elevation (which is not present in reality) and also the trees were not picked-up in the photogrammetric reconstruction. An hypothesis explaining such

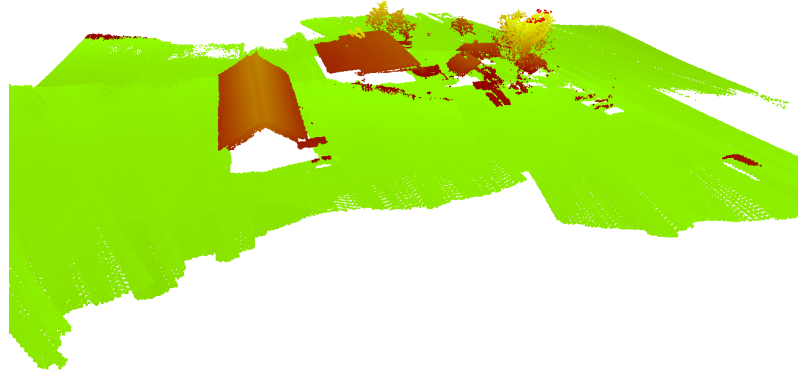

(a) Surface model generated with the LiDAR systems.

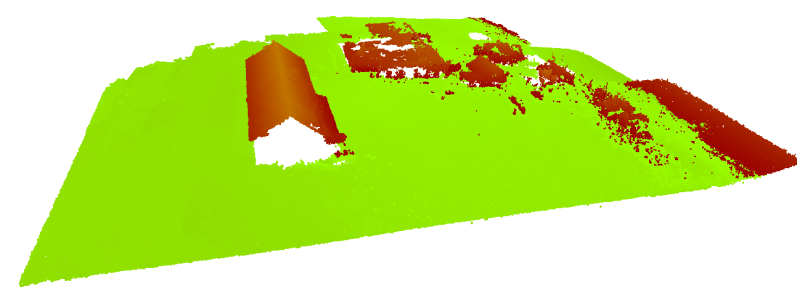

(b) Surface model generated from photogrammetic software.

Figure 5: Digital surface model of the airfield.

a problem is that $40 \%$ image side overlap is too low for this type of terrain.

In order to give a quantitative evaluation of the model accuracy, the long hangar building (it can be observed at the bottom of Figure 4 (a)) was modeled and used as a reference. The model is shown in Figure 6 and it was built from LiDAR measurements taken from 4 different positions on the ground. Each facade was scanned by changing the LiDAR elevation angle while keeping the position static.

The hangar building was then extracted from the ALS and photogrammetric reconstructions and compared to the reference model. Figure 7 shows the deviation for both cases. Before the deviation was calculated the point clouds have been registered with the reference model using the ICP method (Besl and McKay, 1992). This was done to remove uncompensated biases. CloudCompare software was used to perform the comparison (CloudCompare, 2013). The computed error is the distance between the two point clouds.

\subsection{Multi-echo Capabilities}

The aim of this section is to give a preliminary evaluation of the performance of the multi-echo capability of the SICK LMS511 PRO when operating over a forest. In this context high-end LiDAR systems can directly provide valuable information about forest canopy height, topography, etc. (Pirotti, 2011).

The LiDAR used in this experiment is not originally designed for this kind of application and cannot be directly compared to 


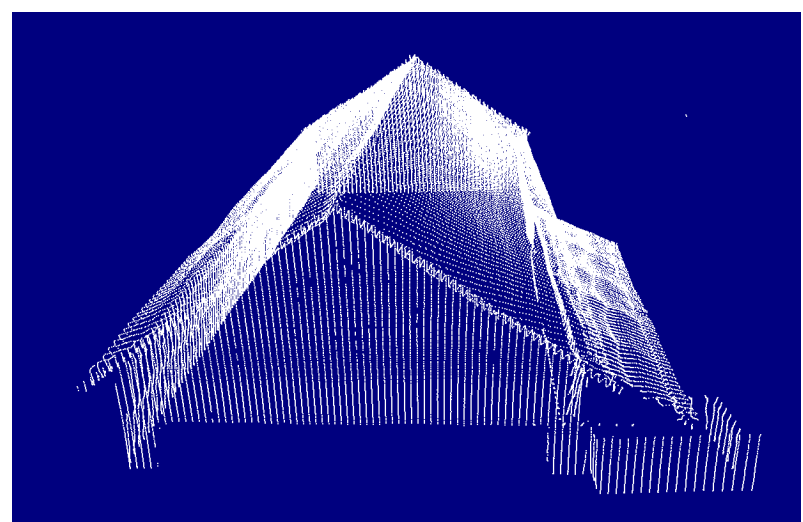

Figure 6: Hangar reference model generated from LiDAR measurements taken from the ground.

high-end airborne LiDAR systems. In any case the capability of recording 5 different echoes for one pulse can improve the performance in estimating the canopy height if compared to a single return LiDAR.

A flight-test was performed over a small forest with canopy height of about 25 meters. The flight altitude was approximately 50 meters from the ground level. All five LiDAR echoes were recorded in the log file. The flight path was executed at the forest border and the measurements falling outside the forest area were manually removed. Figure 8 (a) shows a snapshot of the helicopter camera view over the forest. Figure 8 (b) shows the top view of the LiDAR measurements while in Figure 8 (c) only the groundpoints are displayed. The ground-points are extracted from elevation by thresholding using the flat-ground information. It can be observed that the ground-point distribution at the forest border has higher density (top of the Figure 8 (c)). This is due to two reasons: the area was exactly below the flight path, which means higher density of points collected, and in addition the trees were much shorter when compared to the inner part of the forest.

Figure 9 displays a side view of the LiDAR first echo (a), second echo (b) and third echo (c). The fourth and fifth echoes are not displayed since very few readings were recorded. Table 6.2 presents statistics of the pulse returns of the scanned area. The density of measurement points collected is over 100 per square meter which is very high compared to standard density normally obtained from specialized LiDARs for forest studies (around 10 points per square meter). As can be seen from the data the 4th and 5th echoes did not provide any sensible information due to the low number of returns. This implies that with this setup it is difficult to obtain direct information about the bare earth profile and the tree canopy height without performing specialized data filtering.

\begin{tabular}{|l|rrr|}
\hline Echoes & Total & Ground & \% Ground \\
\hline 1st & 1141195 & 182361 & $16 \%$ \\
2nd & 172507 & 27502 & $16 \%$ \\
3rd & 10233 & 1571 & $15.3 \%$ \\
4th & 241 & 18 & $7.47 \%$ \\
5th & 3 & 3 & $100 \%$ \\
all & 1324179 & 211455 & $16 \%$ \\
\hline
\end{tabular}

Table 2: Number of echoes and classified ground-points for the scanned forest area of 1 hectare size.

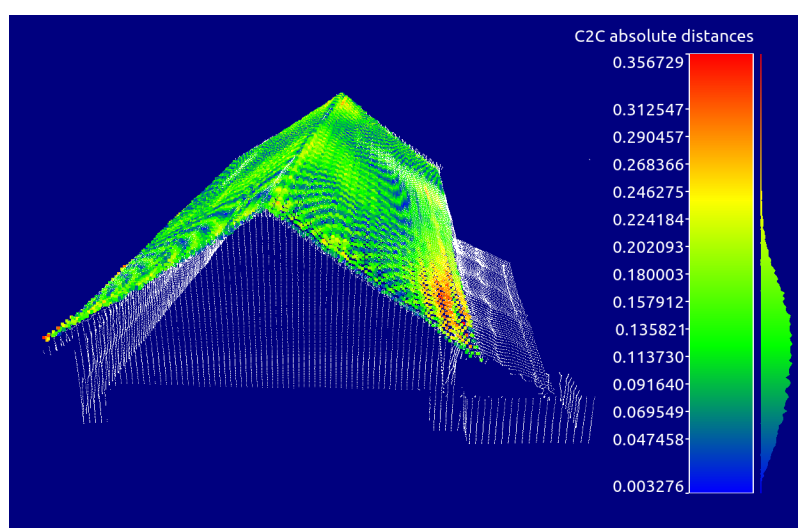

(a) Deviation between the airborne LiDAR reconstruction and the reference model. The standard deviation error is 0.048 meters (distance between the two point clouds).

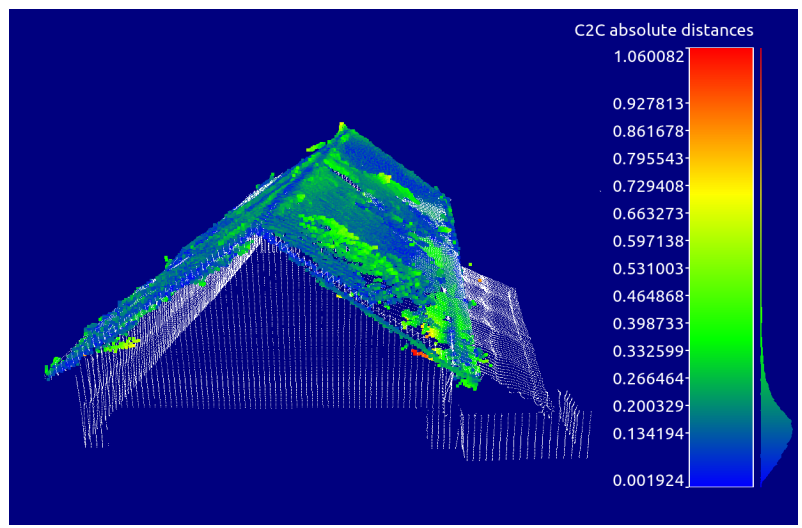

(b) Deviation between the photogrammetric reconstruction and the reference model. The standard deviation error is 0.09 meters.

Figure 7: Deviation between the reference model and reconstruction based on the two methods examined. The values of on the color bar are in meters. On the right side of each color bar the histogram of the error distribution is presented.
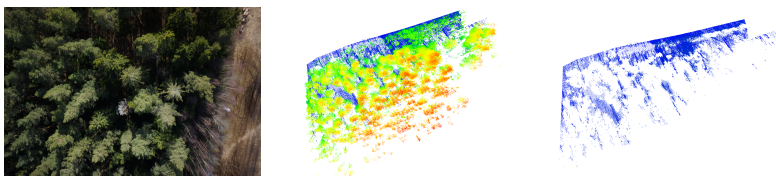

(a) Helicopter camera (b) LiDAR scanning (c) Classified ground view. $\quad$ top view (all 5 echoes). points top view (all 5 echoes).

Figure 8: Camera image and LiDAR measurements top view.

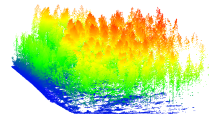

(a) First echo

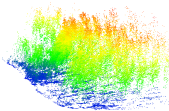

(b) Second echo.

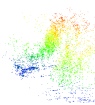

(c) Third echo.
Figure 9: LiDAR echoes plots.

\section{CONCLUSIONS}

The ALS system presented here is suitable for generating dense and accurate digital surface models. Centimeter level accuracy has been achieved in the generated surface model. Low cost MEMS inertial measurement units can be used for this purpose.

The surface model generated with the LiDAR system has been compared with the model generated with a photogrammetric ap- 
proach using a commercial software named Pix4UAV. A building structure was accurately modeled beforehand and used as a reference to evaluate the errors of the two methods. The photogrammetric reconstruction shows a deviation error of $9 \mathrm{~cm}$ against the $4.8 \mathrm{~cm}$ of the LiDAR system. In addition the photogrammetric mapping approach requires a more careful flight planning compared to the LiDAR system. On the other hand, a photogrammetric mapping system based on consumer cameras is less complex, cheaper and lighter and therefore is often the preferred choice in many light weight applications.

\subsection{Acknowledgements}

This work is partially supported by the EU FP7 project SHERPA (grant agreement 600958), the Swedish Research Council (VR) Linnaeus Center for Control, Autonomy, and Decision- making in Complex Systems (CADICS), the ELLIIT network organization for Information and Communication Technology, the Swedish National Aviation Engineering Research Program NFFP5 and the SSF the Swedish Foundation for Strategic Research (CUAS Project).

\section{REFERENCES}

Besl, P. and McKay, N. D., 1992. A method for registration of 3-d shapes. Pattern Analysis and Machine Intelligence, IEEE Transactions on 14(2), pp. 239-256.

CloudCompare, 2013. (version 2.4) [gpl software]. http://www.danielgm.net/cc/.

Conte, G., 2009. Vision-Based Localization and Guidance for Unmanned Aerial Vehicles. PhD thesis, Linköping University, Sweden.

Doherty, P., Haslum, P., Heintz, F., Merz, T., Persson, T. and Wingman, B., 2004. A Distributed Architecture for Autonomous Unmanned Aerial Vehicle Experimentation. In: Proc. of the Int. Symp. on Distributed Autonomous Robotic Systems, pp. 221230.

Glennie, C., Brooks, B., Ericksen, T., Hauser, D., Hudnut, K., Foster, J. and Avery, J., 2013. Compact multipurpose mobile laser scanning system - initial tests and results. Remote Sensing 5(2), pp. 521-538.

Habib, A. F., Kersting, A. P., Ruifang, Z., Al-Durgham, M., Kim, C. and C., L. D., 2008. Lidar strip adjustment using conjugate linear features in overlapping strips. The International Archives of the Photogrammetry, Remote Sensing and Spatial Information Sciences.

Huising, E. J. and Pereira, L. M. G., 1998. Errors and accuracy estimates of laser data acquired by various laser scanning systems for topographic applications. ISPRS Journal of Photogrammetry and Remote Sensing.

Kaestner, R., Thrun, S., Montemerlo, M. and Whalley, M., 2006. A non-rigid approach to scan alignment and change detection using range sensor data. In: Field and Service Robotics, Springer, pp. $179-194$.

Lin, Y., Hyyppa, J. and Jaakkola, A., 2011. Mini-uav-borne lidar for fine-scale mapping. Geoscience and Remote Sensing Letters, IEEE 8(3), pp. 426-430.

Marconi, L., Melchiorri, C., Beetz, M., Pangercic, D., Siegwart, R., Leutenegger, S., Carloni, R., Stramigioli, S., Bruyninckx, H., Doherty, P., Kleiner, A., Lippiello, V., Finzi, A., Siciliano, B., Sala, A. and Tomatis, N., 2012. The sherpa project: smart collaboration between humans and ground-aerial robots for improving rescuing activities in alpine environments. In: IEEE International Symposium on Safety, Security, and Rescue Robotics (SSRR), College Station, Texas, USA.
Pirotti, F., 2011. Analysis of full-waveform lidar data for forestry applications: a review of investigations and methods. iForest Biogeosciences and Forestry (3), pp. 100-106.

Pix4d, 2013. Pix4uav desktop 3d (version 2.1). http://pix4d.com/.

Rauch, H., Tung, F. and Striebel, C., 1965. Maximum likelihood estimates of linear dynamic systems. AIAA Journal 3(8), pp. 1445-1450.

Skaloud, J., 1999. Optimizing Georeferencing of Airborne Survey Systems by INS/DGPS. PhD thesis, University of Calgary, Alberta.

Skaloud, J. and Lichti, D., 2006. Rigorous approach to boresight self-calibration in airborne laser scanning. ISPRS journal of photogrammetry and remote sensing 61(1), pp. 47-59.

Vallet, J., 2002. Saisie de la couverture neigeuse de sites avalancheux par des systèmes aéroportés. $\mathrm{PhD}$ thesis, $\mathrm{Ph}$. D. Thèse $n$.

Vallet, J., 2008. High precision lidar mapping for complex mountain topography. In: 6th ICA Mountain Cartography Workshop Mountain Mapping and Visualization, Lenk, Swizerland.

Williams, S., Parker, L. T. and Howard, A. M., 2012. Terrain reconstruction of glacial surfaces. IEEE Robotics and Automation Magazine pp. 59-71. 